\title{
GRADIENT CONVERGENCE IN GRADIENT METHODS WITH ERRORS*
}

\author{
DIMITRI P. BERTSEKAS ${ }^{\dagger}$ AND JOHN N. TSITSIKLIS ${ }^{\dagger}$
}

\begin{abstract}
We consider the gradient method $x_{t+1}=x_{t}+\gamma_{t}\left(s_{t}+w_{t}\right)$, where $s_{t}$ is a descent direction of a function $f: \Re^{n} \rightarrow \Re$ and $w_{t}$ is a deterministic or stochastic error. We assume that $\nabla f$ is Lipschitz continuous, that the stepsize $\gamma_{t}$ diminishes to 0 , and that $s_{t}$ and $w_{t}$ satisfy standard conditions. We show that either $f\left(x_{t}\right) \rightarrow-\infty$ or $f\left(x_{t}\right)$ converges to a finite value and $\nabla f\left(x_{t}\right) \rightarrow 0$ (with probability 1 in the stochastic case), and in doing so, we remove various boundedness conditions that are assumed in existing results, such as boundedness from below of $f$, boundedness of $\nabla f\left(x_{t}\right)$, or boundedness of $x_{t}$.
\end{abstract}

Key words. gradient methods, incremental gradient methods, stochastic approximation, gradient convergence

AMS subject classifications. 62L20, 903C0

PII. S1052623497331063

1. Introduction. We consider the problem

$$
\begin{array}{ll}
\operatorname{minimize} & f(x) \\
\text { subject to } & x \in \Re^{n},
\end{array}
$$

where $\Re^{n}$ denotes the $n$-dimensional Euclidean space and $f: \Re^{n} \mapsto \Re$ is a continuously differentiable function, such that for some constant $L$ we have

$$
\|\nabla f(x)-\nabla f(\bar{x})\| \leq L\|x-\bar{x}\| \quad \forall x, \bar{x} \in \Re^{n} .
$$

The purpose of this paper is to sharpen the existing convergence theory for the classical descent method

$$
x_{t+1}=x_{t}+\gamma_{t}\left(s_{t}+w_{t}\right)
$$

where

(a) $\gamma_{t}$ is a positive stepsize sequence satisfying

$$
\sum_{t=0}^{\infty} \gamma_{t}=\infty, \quad \sum_{t=0}^{\infty} \gamma_{t}^{2}<\infty
$$

(b) $s_{t}$ is a descent direction satisfying for some positive scalars $c_{1}$ and $c_{2}$, and all $t$,

$$
c_{1}\left\|\nabla f\left(x_{t}\right)\right\|^{2} \leq-\nabla f\left(x_{t}\right)^{\prime} s_{t}, \quad\left\|s_{t}\right\| \leq c_{2}\left\|\nabla f\left(x_{t}\right)\right\|
$$

(c) $w_{t}$ either is a deterministic error satisfying for some positive scalars $p$ and $q$, and all $t$,

$$
\left\|w_{t}\right\| \leq \gamma_{t}\left(q+p\left\|\nabla f\left(x_{t}\right)\right\|\right)
$$

* Received by the editors December 4, 1997; accepted for publication (in revised form) May 24, 1999; published electronically February 29, 2000. This work was supported by NSF under grant DMI-9625489.

http://www.siam.org/journals/siopt/10-3/33106.html

${ }^{\dagger}$ Department of Electrical Engineering and Computer Science, MIT, Cambridge, MA 02139 (dimitrib@mit.edu, jnt@mit.edu). 
or is a stochastic error satisfying conditions that are standard in stochastic gradient and stochastic approximation methods.

Our main result is that either $f\left(x_{t}\right) \rightarrow-\infty$ or $f\left(x_{t}\right)$ converges to a finite value and $\lim _{t \rightarrow \infty} \nabla f\left(x_{t}\right)=0$ (with probability 1 on the stochastic case).

The method where the errors $w_{t}$ are deterministic includes as a special case the standard incremental gradient/backpropagation method for neural network training, the convergence of which has been the object of much recent analysis [Luo91], [Gai94], [Gri94], [LuT94], [MaS94], [Man93], [Ber95a] (see [BeT96] for our discussion of incremental gradient methods and their application to neural network training). The method where the errors $w_{t}$ are stochastic includes as a special case the classical Robbins-Monro/stochastic gradient method, as well as methods involving scaling of the gradient and satisfying the pseudogradient condition of Poljak and Tsypkin [PoT73]; see section 4 for a precise statement of our assumptions. Basically, the entire spectrum of unconstrained gradient methods is considered, with the only restriction being the diminishing stepsize condition (1.4) (which is essential for convergence in gradient methods with errors) and the attendant Lipschitz condition (1.2) (which is necessary for showing any kind of convergence result under the stepsize condition $(1.4))$.

To place our analysis in perspective, we review the related results of the literature for gradient-like methods with errors and in the absence of convexity. Our results relate to two types of analysis:

(1) Results that are based on some type of deterministic or stochastic descent argument, such as the use of a Lyapunov function or a supermartingale convergence theorem. All of the results of this type known to us assume that $f$ is bounded below and in some cases require a boundedness assumption on the sequence $\left\{x_{t}\right\}$ or show only that $\liminf _{t \rightarrow \infty}\left\|\nabla f\left(x_{t}\right)\right\|=0$. By contrast, we show that $\lim _{t \rightarrow \infty}\left\|\nabla f\left(x_{t}\right)\right\|=0$ and we also deal with the case where $f$ is unbounded below and $\left\{x_{t}\right\}$ is unbounded. In fact, a principal aim of our work has been to avoid any type of boundedness assumption. For example, the classical analysis of Poljak and Tsypkin [PoT73], under essentially the same conditions as ours, shows that if $f$ is bounded below, then $f\left(x_{t}\right)$ converges and $\liminf \operatorname{in}_{t \rightarrow \infty}\left\|\nabla f\left(x_{t}\right)\right\|=0$ (see Poljak [Pol87, p. 51]). The analysis of Gaivoronski [Gai94], for stochastic gradient and incremental gradient methods, under similar conditions to ours shows that $\lim _{t \rightarrow \infty}\left\|\nabla f\left(x_{t}\right)\right\|=0$, but it also assumes that $f(x)$ is bounded below and that $\|\nabla f(x)\|$ is bounded over $\Re^{n}$. The analysis of Luo and Tseng [LuT94] for the incremental gradient method shows that $\lim _{t \rightarrow \infty}\left\|\nabla f\left(x_{t}\right)\right\|=0$, but it also assumes that $f(x)$ is bounded below, and it makes some additional assumptions on the stepsize $\gamma_{t}$. The analyses by Grippo [Gri94] and by Mangasarian and Solodov [MaS94] for the incremental gradient method (with and without a momentum term) make assumptions that are different from ours and include boundedness of the generated sequence $x_{t}$. The analysis of Walk [Wal92, p. 2] (see also Pflug [Pfl96, p. 282]) shows that $\lim _{t \rightarrow \infty}\left\|\nabla f\left(x_{t}\right)\right\|=0$, assuming that $s_{t}=-\nabla f\left(x_{t}\right)$, that $w_{t}$ is deterministic and satisfies somewhat different conditions than ours, and that $f$ is bounded below. Our method of proof for the case of deterministic errors is similar to the method of Walk. (The assumption that $f$ is bounded below is not critical for Walk's analysis.) However, in the case of stochastic errors, standard stochastic descent proofs rely critically on the boundedness of $f$ from below, and we have used a new line of proof for our result (see the discussion in section 4).

(2) Results based on the so-called ODE analysis [Lju77], [KuC78], [BMP90], [KuY97] that relate the evolution of the algorithm to the trajectories of a differ- 
ential equation $d x / d t=h(x)$. For example, if we are dealing with the stochastic steepest descent method $x_{t+1}=x_{t}-\gamma_{t}\left(\nabla f\left(x_{t}\right)-w_{t}\right)$, the corresponding ODE is $d x / d t=-\nabla f(x)$. This framework typically involves an explicit or implicit assumption that the average direction of update $h(x)$ is a well-defined function of the current iterate $x$. It cannot be applied, for example, to a gradient method with diagonal scaling, where the scaling may depend in a complicated way on the past history of the algorithm, unless one works with differential inclusions - rather than differential equations - for which not many results are available. For another example, an asynchronous gradient iteration that updates a single component at a time (selected by some arbitrary or hard-to-model mechanism) does not lead to a well-defined average direction of update $h(x)$, unless one makes some very special assumptions, e.g., the stepsize assumptions of Borkar [Bor95]. In addition to the above described difficulty, the ODE approach relies on the assumption that the sequence of iterates $x_{t}$ is bounded or recurrent, something that must be independently verified. Let us also mention the following more recent results by Delyon [Del96], which have some similarities with ours: they are proved using a potential function argument and can establish the convergence of $\nabla f\left(x_{t}\right)$ to zero. Similar to the ODE approach, these results assume a well-defined average update direction $h(x)$ and are based on boundedness or recurrence assumptions.

The paper is organized as follows. In the next section, we focus on the method where there is a nonrandom error $w_{t}$ satisfying the condition (1.6). The convergence result obtained is then applied in section 3 to the case of incremental gradient methods for minimizing the sum of a large number of functions. In section 4 , we focus on stochastic gradient methods. Finally, in section 5, a stochastic version of the incremental gradient method is discussed.

2. Deterministic gradient methods with errors. Throughout the paper, we focus on the unconstrained minimization of a continuously differentiable function $f: \Re^{n} \mapsto \Re$, satisfying for some constant $L$

$$
\|\nabla f(x)-\nabla f(\bar{x})\| \leq L\|x-\bar{x}\| \quad \forall x, \bar{x} \in \Re^{n} .
$$

As mentioned in the preceding section, the line of proof of the following proposition is known, although some of our assumptions differ slightly from those in the literature. We will need the following known lemma, which we prove for completeness.

Lemma 1. Let $Y_{t}, W_{t}$, and $Z_{t}$ be three sequences such that $W_{t}$ is nonnegative for all t. Assume that

$$
Y_{t+1} \leq Y_{t}-W_{t}+Z_{t}, \quad t=0,1, \ldots,
$$

and that the series $\sum_{t=0}^{T} Z_{t}$ converges as $T \rightarrow \infty$. Then either $Y_{t} \rightarrow-\infty$ or else $Y_{t}$ converges to a finite value and $\sum_{t=0}^{\infty} W_{t}<\infty$.

Proof. Let $\bar{t}$ be any nonnegative integer. By adding the relation $Y_{t+1} \leq Y_{t}+Z_{t}$ over all $t \geq \bar{t}$ and by taking the limit superior as $t \rightarrow \infty$, we obtain

$$
\limsup _{t \rightarrow \infty} Y_{t} \leq Y_{\bar{t}}+\sum_{t=\bar{t}}^{\infty} Z_{t}<\infty .
$$

By taking the limit inferior of the right-hand side as $\bar{t} \rightarrow \infty$ and using the fact $\lim _{\bar{t} \rightarrow \infty} \sum_{t=\bar{t}}^{\infty} Z_{t}=0$, we obtain

$$
\limsup _{t \rightarrow \infty} Y_{t} \leq \liminf _{\bar{t} \rightarrow \infty} Y_{\bar{t}}<\infty
$$


This implies that either $Y_{t} \rightarrow-\infty$ or else $Y_{t}$ converges to a finite value. In the latter case, by adding the relation $Y_{i+1} \leq Y_{i}-W_{i}+Z_{i}$ from $i=0$ to $i=t$, we obtain

$$
\sum_{i=0}^{t} W_{i} \leq Y_{0}+\sum_{i=0}^{t} Z_{i}-Y_{t+1}, \quad t=0,1, \ldots,
$$

which implies that $\sum_{i=0}^{\infty} W_{i} \leq Y_{0}+\sum_{i=0}^{\infty} Z_{i}-\lim _{t \rightarrow \infty} Y_{t}<\infty$.

We have the following result.

Proposition 1. Let $x_{t}$ be a sequence generated by the method

$$
x_{t+1}=x_{t}+\gamma_{t}\left(s_{t}+w_{t}\right)
$$

where $s_{t}$ is a descent direction satisfying for some positive scalars $c_{1}$ and $c_{2}$, and all $t$,

$$
c_{1}\left\|\nabla f\left(x_{t}\right)\right\|^{2} \leq-\nabla f\left(x_{t}\right)^{\prime} s_{t}, \quad\left\|s_{t}\right\| \leq c_{2}\left(1+\left\|\nabla f\left(x_{t}\right)\right\|\right),
$$

and $w_{t}$ is an error vector satisfying for some positive scalars $p$ and $q$, and all $t$,

$$
\left\|w_{t}\right\| \leq \gamma_{t}\left(q+p\left\|\nabla f\left(x_{t}\right)\right\|\right)
$$

Assume that the stepsize $\gamma_{t}$ is positive and satisfies

$$
\sum_{t=0}^{\infty} \gamma_{t}=\infty, \quad \sum_{t=0}^{\infty} \gamma_{t}^{2}<\infty .
$$

Then either $f\left(x_{t}\right) \rightarrow-\infty$ or else $f\left(x_{t}\right)$ converges to a finite value and $\lim _{t \rightarrow \infty} \nabla f\left(x_{t}\right)=$ 0. Furthermore, every limit point of $x_{t}$ is a stationary point of $f$.

Proof. Fix two vectors $x$ and $z$, let $\xi$ be a scalar parameter, and let $g(\xi)=$ $f(x+\xi z)$. The chain rule yields $(d g / d \xi)(\xi)=z^{\prime} \nabla f(x+\xi z)$. We have

$$
\begin{aligned}
f(x+z)-f(x) & =g(1)-g(0) \\
& =\int_{0}^{1} \frac{d g}{d \xi}(\xi) d \xi \\
& =\int_{0}^{1} z^{\prime} \nabla f(x+\xi z) d \xi \\
& \leq \int_{0}^{1} z^{\prime} \nabla f(x) d \xi+\left|\int_{0}^{1} z^{\prime}(\nabla f(x+\xi z)-\nabla f(x)) d \xi\right| \\
& \leq z^{\prime} \nabla f(x)+\int_{0}^{1}\|z\| \cdot\|\nabla f(x+\xi z)-\nabla f(x)\| d \xi \\
& \leq z^{\prime} \nabla f(x)+\|z\| \int_{0}^{1} L \xi\|z\| d \xi \\
& =z^{\prime} \nabla f(x)+\frac{L}{2}\|z\|^{2} .
\end{aligned}
$$

We apply (2.4) with $x=x_{t}$ and $z=\gamma_{t}\left(s_{t}+w_{t}\right)$. We obtain

$$
f\left(x_{t+1}\right) \leq f\left(x_{t}\right)+\gamma_{t} \nabla f\left(x_{t}\right)^{\prime}\left(s_{t}+w_{t}\right)+\frac{\gamma_{t}^{2} L}{2}\left\|s_{t}+w_{t}\right\|^{2} .
$$


Using our assumptions, we have

$$
\begin{aligned}
\nabla f\left(x_{t}\right)^{\prime}\left(s_{t}+w_{t}\right) & \leq-c_{1}\left\|\nabla f\left(x_{t}\right)\right\|^{2}+\left\|\nabla f\left(x_{t}\right)\right\|\left\|w_{t}\right\| \\
& \leq-c_{1}\left\|\nabla f\left(x_{t}\right)\right\|^{2}+\gamma_{t} q\left\|\nabla f\left(x_{t}\right)\right\|+\gamma_{t} p\left\|\nabla f\left(x_{t}\right)\right\|^{2} .
\end{aligned}
$$

Furthermore, using the relations $\left\|s_{t}\right\|^{2} \leq 2 c_{2}^{2}\left(1+\left\|\nabla f\left(x_{t}\right)\right\|^{2}\right)$ and $\left\|w_{t}\right\|^{2} \leq 2 \gamma_{t}^{2}\left(q^{2}+\right.$ $\left.p^{2}\left\|\nabla f\left(x_{t}\right)\right\|^{2}\right)$, which follow from (2.2) and (2.3), respectively, we have

$$
\begin{aligned}
\left\|s_{t}+w_{t}\right\|^{2} & \leq 2\left\|s_{t}\right\|^{2}+2\left\|w_{t}\right\|^{2} \\
& \leq 4 c_{2}^{2}\left(1+\left\|\nabla f\left(x_{t}\right)\right\|^{2}\right)+4 \gamma_{t}^{2}\left(q^{2}+p^{2}\left\|\nabla f\left(x_{t}\right)\right\|^{2}\right) .
\end{aligned}
$$

Combining the above relations, we obtain

$$
\begin{gathered}
f\left(x_{t+1}\right) \leq f\left(x_{t}\right)-\gamma_{t}\left(c_{1}-\gamma_{t} p-2 \gamma_{t} c_{2}^{2} L-2 \gamma_{t}^{3} p^{2} L\right)\left\|\nabla f\left(x_{t}\right)\right\|^{2} \\
+\gamma_{t}^{2} q\left\|\nabla f\left(x_{t}\right)\right\|+2 \gamma_{t}^{2} c_{2}^{2} L+2 \gamma_{t}^{4} q^{2} L .
\end{gathered}
$$

Since $\gamma_{t} \rightarrow 0$, we have for some positive constant $c$ and all $t$ sufficiently large

$$
f\left(x_{t+1}\right) \leq f\left(x_{t}\right)-\gamma_{t} c\left\|\nabla f\left(x_{t}\right)\right\|^{2}+\gamma_{t}^{2} q\left\|\nabla f\left(x_{t}\right)\right\|+2 \gamma_{t}^{2} c_{2}^{2} L+2 \gamma_{t}^{4} q^{2} L .
$$

Using the inequality $\left\|\nabla f\left(x_{t}\right)\right\| \leq 1+\left\|\nabla f\left(x_{t}\right)\right\|^{2}$, the above relation yields for all $t$

$$
f\left(x_{t+1}\right) \leq f\left(x_{t}\right)-\gamma_{t}\left(c-\gamma_{t} q\right)\left\|\nabla f\left(x_{t}\right)\right\|^{2}+\gamma_{t}^{2}\left(q+2 c_{2}^{2} L\right)+2 \gamma_{t}^{4} q^{2} L,
$$

which for sufficiently large $t$ can be written as

$$
f\left(x_{t+1}\right) \leq f\left(x_{t}\right)-\gamma_{t} \beta_{1}\left\|\nabla f\left(x_{t}\right)\right\|^{2}+\gamma_{t}^{2} \beta_{2},
$$

where $\beta_{1}$ and $\beta_{2}$ are some positive scalars.

By using (2.5), Lemma 1, and the assumption $\sum_{t=0}^{\infty} \gamma_{t}^{2}<\infty$, we see that either $f\left(x_{t}\right) \rightarrow-\infty$ or else $f\left(x_{t}\right)$ converges and

$$
\sum_{t=0}^{\infty} \gamma_{t}\left\|\nabla f\left(x_{t}\right)\right\|^{2}<\infty
$$

If there existed an $\epsilon>0$ and an integer $\bar{t}$ such that $\left\|\nabla f\left(x_{t}\right)\right\| \geq \epsilon$ for all $t \geq \bar{t}$, we would have

$$
\sum_{t=\bar{t}}^{\infty} \gamma_{t}\left\|\nabla f\left(x_{t}\right)\right\|^{2} \geq \epsilon^{2} \sum_{t=\bar{t}}^{\infty} \gamma_{t}=\infty
$$

which contradicts (2.6). Therefore, $\liminf _{t \rightarrow \infty}\left\|\nabla f\left(x_{t}\right)\right\|=0$.

To show that $\lim _{t \rightarrow \infty} \nabla f\left(x_{t}\right)=0$, assume the contrary; that is, $\lim \sup _{t \rightarrow \infty}$ $\left\|\nabla f\left(x_{t}\right)\right\|>0$. Then there exists an $\epsilon>0$ such that $\left\|\nabla f\left(x_{t}\right)\right\|<\epsilon / 2$ for infinitely many $t$ and also $\left\|\nabla f\left(x_{t}\right)\right\|>\epsilon$ for infinitely many $t$. Therefore, there is an infinite subset of integers $\mathcal{T}$ such that for each $t \in \mathcal{T}$, there exists an integer $i(t)>t$ such that

$$
\begin{aligned}
& \left\|\nabla f\left(x_{t}\right)\right\|<\epsilon / 2, \quad\left\|\nabla f\left(x_{i(t)}\right)\right\|>\epsilon, \\
& \epsilon / 2 \leq\left\|\nabla f\left(x_{i}\right)\right\| \leq \epsilon \quad \text { if } t<i<i(t) .
\end{aligned}
$$


Since

$$
\begin{aligned}
\left\|\nabla f\left(x_{t+1}\right)\right\|-\left\|\nabla f\left(x_{t}\right)\right\| & \leq\left\|\nabla f\left(x_{t+1}\right)-\nabla f\left(x_{t}\right)\right\| \\
& \leq L\left\|x_{t+1}-x_{t}\right\| \\
& =\gamma_{t} L\left\|s_{t}\right\| \\
& \leq \gamma_{t} L c_{2}\left(1+\left\|\nabla f\left(x_{t}\right)\right\|\right),
\end{aligned}
$$

it follows that for all $t \in \mathcal{T}$ that are sufficiently large so that $\gamma_{t} L c_{2}<\epsilon / 4$, we have

$$
\epsilon / 4 \leq\left\|\nabla f\left(x_{t}\right)\right\|
$$

otherwise, the condition $\epsilon / 2 \leq\left\|\nabla f\left(x_{t+1}\right)\right\|$ would be violated. Without loss of generality, we assume that the above relations as well as (2.5) hold for all $t \in \mathcal{T}$.

We have for all $t \in \mathcal{T}$, using the condition $\left\|s_{t}\right\| \leq c_{2}\left(1+\left\|\nabla f\left(x_{t}\right)\right\|\right)$ and the Lipschitz condition (2.1),

$$
\begin{aligned}
\frac{\epsilon}{2} & \leq\left\|\nabla f\left(x_{i(t)}\right)\right\|-\left\|\nabla f\left(x_{t}\right)\right\| \\
& \leq\left\|\nabla f\left(x_{i(t)}\right)-\nabla f\left(x_{t}\right)\right\| \\
& \leq L\left\|x_{i(t)}-x_{t}\right\| \\
& \leq L \sum_{i=t}^{i(t)-1} \gamma_{i}\left(\left\|s_{i}\right\|+\left\|w_{i}\right\|\right) \\
& \leq L c_{2} \sum_{i=t}^{i(t)-1} \gamma_{i}\left(1+\left\|\nabla f\left(x_{i}\right)\right\|\right)+L \sum_{i=t}^{i(t)-1} \gamma_{i}^{2}\left(q+p\left\|\nabla f\left(x_{i}\right)\right\|\right) \\
& \leq L c_{2}(1+\epsilon) \sum_{i=t}^{i(t)-1} \gamma_{i}+L(q+p \epsilon) \sum_{i=t}^{i(t)-1} \gamma_{i}^{2} .
\end{aligned}
$$

From this it follows that

$$
\frac{1}{2 L c_{2}(1+\epsilon)} \leq \liminf _{t \rightarrow \infty} \sum_{i=t}^{i(t)-1} \gamma_{i}
$$

Using (2.5), we see that

$$
f\left(x_{i(t)}\right) \leq f\left(x_{t}\right)-\beta_{1}\left(\frac{\epsilon}{4}\right)^{2} \sum_{i=t}^{i(t)-1} \gamma_{i}+\beta_{2} \sum_{i=t}^{i(t)-1} \gamma_{i}^{2} \quad \forall t \in \mathcal{T} .
$$

Using the convergence of $f\left(x_{t}\right)$ already shown and the assumption $\sum_{t=0}^{\infty} \gamma_{t}^{2}<\infty$, this relation implies that

$$
\lim _{t \rightarrow \infty, t \in \mathcal{T}} \sum_{i=t}^{i(t)-1} \gamma_{i}=0
$$

and contradicts (2.8).

Finally, if $\bar{x}$ is a limit point of $x_{t}$, then $f\left(x_{t}\right)$ converges to the finite value $f(\bar{x})$. Thus we have $\nabla f\left(x_{t}\right) \rightarrow 0$, implying that $\nabla f(\bar{x})=0$. 
3. Incremental gradient methods. In this section, we apply the results of the preceding section to the case where $f$ has the form

$$
f(x)=\sum_{i=1}^{m} f_{i}(x)
$$

where $f_{i}: \Re^{n} \mapsto \Re$ is for every $i$ a continuously differentiable function satisfying the Lipschitz condition

$$
\left\|\nabla f_{i}(x)-\nabla f_{i}(\bar{x})\right\| \leq L\|x-\bar{x}\| \quad \forall x, \bar{x} \in \Re^{n}
$$

for some constant $L$.

In situations where there are many component functions $f_{i}$, it may be attractive to use an incremental method that does not wait to process the entire set of components before updating $x$; instead, the method cycles through the components in sequence and updates the estimate of $x$ after each component is processed. In particular, given $x_{t}$, we may obtain $x_{t+1}$ as

$$
x_{t+1}=\psi_{m},
$$

where $\psi_{m}$ is obtained at the last step of the algorithm

$$
\psi_{i}=\psi_{i-1}-\gamma_{t} \nabla f_{i}\left(\psi_{i-1}\right), \quad i=1, \ldots, m,
$$

and

$$
\psi_{0}=x_{t} .
$$

This method can be written as

$$
x_{t+1}=x_{t}-\gamma_{t} \sum_{i=1}^{m} \nabla f_{i}\left(\psi_{i-1}\right) .
$$

It is referred to as the incremental gradient method, and it is used extensively in the training of neural networks. It should be compared with the ordinary gradient method, which is

$$
x_{t+1}=x_{t}-\gamma_{t} \nabla f\left(x_{t}\right)=x_{t}-\gamma_{t} \sum_{i=1}^{m} \nabla f_{i}\left(x_{t}\right) .
$$

Thus, a cycle of the incremental gradient method through the components $f_{i}$ differs from an ordinary gradient iteration only in that the evaluation of $\nabla f_{i}$ is done at the corresponding current estimates $\psi_{i-1}$ rather than at the estimate $x_{t}$ available at the start of the cycle. The advantages of incrementalism in enhancing the speed of convergence (at least in the early stages of the method) are well known; see, for example, the discussions in [Ber95a], [Ber95b], [BeT96].

The main idea of the following convergence proof is that the incremental gradient method can be viewed as the regular gradient iteration where the gradient is perturbed by an error term that is proportional to the stepsize. In particular, if we compare the incremental method (3.4) with the ordinary gradient method (3.5), we see that the error term in the gradient direction is bounded by

$$
\sum_{i=1}^{m}\left\|\nabla f_{i}\left(\psi_{i-1}\right)-\nabla f_{i}\left(x_{t}\right)\right\|
$$


In view of our Lipschitz assumption (3.1), this term is bounded by

$$
L \sum_{i=1}^{m}\left\|\psi_{i-1}-x_{t}\right\|
$$

which from (3.2) is seen to be proportional to $\gamma_{t}$. (A more precise argument is given below.)

Proposition 2. Let $x_{t}$ be a sequence generated by the incremental gradient method (3.2)-(3.4). Assume that for some positive constants $C$ and $D$, and all $i=$ $1, \ldots, m$, we have

$$
\left\|\nabla f_{i}(x)\right\| \leq C+D\|\nabla f(x)\| \quad \forall x \in \Re^{n} .
$$

Assume also that

$$
\sum_{t=0}^{\infty} \gamma_{t}=\infty, \quad \sum_{t=0}^{\infty} \gamma_{t}^{2}<\infty .
$$

Then either $f\left(x_{t}\right) \rightarrow-\infty$ or else $f\left(x_{t}\right)$ converges to a finite value and $\lim _{t \rightarrow \infty} \nabla f\left(x_{t}\right)=$ 0 . Furthermore, every limit point of $x_{t}$ is a stationary point of $f$.

Proof. We formulate the incremental gradient method as a gradient method with errors that are proportional to the stepsize and then apply Proposition 1. For simplicity we will assume that there are only two functions $f_{i}$, that is, $m=2$. The proof is similar when $m>2$. We have

$$
\begin{gathered}
\psi_{1}=x_{t}-\gamma_{t} \nabla f_{1}\left(x_{t}\right), \\
x_{t+1}=\psi_{1}-\gamma_{t} \nabla f_{2}\left(\psi_{1}\right) .
\end{gathered}
$$

By adding these two relations, we obtain

$$
x_{t+1}=x_{t}+\gamma_{t}\left(-\nabla f\left(x_{t}\right)+w_{t}\right),
$$

where

$$
w_{t}=\nabla f_{2}\left(x_{t}\right)-\nabla f_{2}\left(\psi_{1}\right)
$$

We have

$$
\left\|w_{t}\right\| \leq L\left\|x_{t}-\psi_{1}\right\|=\gamma_{t} L\left\|\nabla f_{1}\left(x_{t}\right)\right\| \leq \gamma_{t}\left(L C+L D\left\|\nabla f\left(x_{t}\right)\right\|\right) .
$$

Thus Proposition 1 applies.

Condition (3.6) is guaranteed to hold if each $f_{k}$ is of the form

$$
f_{k}(x)=x^{\prime} Q_{k} x+g_{k}^{\prime} x+h_{k},
$$

where each $Q_{k}$ is a positive semidefinite matrix, each $g_{k}$ is a vector, and each $h_{k}$ is a scalar. (This is the generic situation encountered in linear least squares problems.) If $\sum_{k=1}^{K} Q_{k}$ is positive definite, there exists a unique minimum to which the algorithm must converge. In the absence of positive definiteness, we obtain $\nabla f\left(x_{t}\right) \rightarrow 0$ if the optimal cost is finite. If, on the other hand, the optimal cost is $-\infty$, it can be shown that $\|\nabla f(x)\| \geq \alpha$ for some $\alpha>0$ and for all $x$. This implies that $f(x) \rightarrow-\infty$ and that $\|x\| \rightarrow \infty$. 
4. Stochastic gradient methods. In this section, we study stochastic gradient methods. Our main result is similar to Proposition 1 except that we let the noise term $w_{t}$ be of a stochastic nature. Once more, we will prove that $f\left(x_{t}\right)$ converges and, if the limit is finite, $\nabla f\left(x_{t}\right)$ converges to 0 . We comment on the technical issues that arise in establishing such a result. The sequence $f\left(x_{t}\right)$ can be shown to be approximately a supermartingale. The variance of the underlying noise is allowed to grow with $\left\|\nabla f\left(x_{t}\right)\right\|$ and therefore can be unbounded. While such unboundedness has been successfully handled in past works on related methods, new complications arise because no lower bound on $f\left(x_{t}\right)$ is assumed. For that reason, the supermartingale convergence theorem cannot be used in a simple manner. Our approach is to show that whenever $\left\|\nabla f\left(x_{t}\right)\right\|$ is large, it remains so for a sufficiently long time interval, guaranteeing a decrease in the value of $f\left(x_{t}\right)$ which is significant and dominates the noise effects.

Proposition 3. Let $x_{t}$ be a sequence generated by the method

$$
x_{t+1}=x_{t}+\gamma_{t}\left(s_{t}+w_{t}\right)
$$

where $\gamma_{t}$ is a deterministic positive stepsize, $s_{t}$ is a descent direction, and $w_{t}$ is a random noise term. Let $\mathcal{F}_{t}$ be an increasing sequence of $\sigma$-fields. We assume the following:

(a) $x_{t}$ and $s_{t}$ are $\mathcal{F}_{t}$-measurable.

(b) There exist positive scalars $c_{1}$ and $c_{2}$ such that

$$
c_{1}\left\|\nabla f\left(x_{t}\right)\right\|^{2} \leq-\nabla f\left(x_{t}\right) s_{t}, \quad\left\|s_{t}\right\| \leq c_{2}\left(1+\left\|\nabla f\left(x_{t}\right)\right\|\right) \quad \forall t .
$$

(c) We have, for all $t$ and with probability 1 ,

$$
E\left[\left\|w_{t}\right\|^{2} \mid \mathcal{F}_{t}\right] \leq A\left(1+\left\|\nabla f\left(x_{t}\right)\right\|^{2}\right)
$$

where $A$ is a positive deterministic constant.

(d) We have

$$
\sum_{t=0}^{\infty} \gamma_{t}=\infty, \quad \sum_{t=0}^{\infty} \gamma_{t}^{2}<\infty
$$

Then, either $f\left(x_{t}\right) \rightarrow-\infty$ or else $f\left(x_{t}\right)$ converges to a finite value and $\lim _{t \rightarrow \infty}$ $\nabla f\left(x_{t}\right)=0$. Furthermore, every limit point of $x_{t}$ is a stationary point of $f$.

Remarks. (a) The $\sigma$-field $\mathcal{F}_{t}$ should be interpreted as the history of the algorithm up to time $t$, just before $w_{t}$ is generated. In particular, conditioning on $\mathcal{F}_{t}$ can be thought of as conditioning on $x_{0}, s_{0}, w_{0}, \ldots, x_{t-1}, s_{t-1}, w_{t-1}, x_{t}, s_{t}$.

(b) Strictly speaking, the conclusions of the proposition only hold "with probability 1." For simplicity, an explicit statement of this qualification often will be omitted.

(c) Our assumptions on $w_{t}$ are of the same type as those considered in [PoT73]. 
Proof of Proposition 3. We apply (2.4) with $x=x_{t}$ and $z=\gamma_{t}\left(s_{t}+w_{t}\right)$. We obtain

$$
\begin{aligned}
f\left(x_{t+1}\right) \leq & f\left(x_{t}\right)+\gamma_{t} \nabla f\left(x_{t}\right)^{\prime}\left(s_{t}+w_{t}\right)+\frac{\gamma_{t}^{2} L}{2}\left\|s_{t}+w_{t}\right\|^{2} \\
\leq & f\left(x_{t}\right)-\gamma_{t} c_{1}\left\|\nabla f\left(x_{t}\right)\right\|^{2}+\gamma_{t} \nabla f\left(x_{t}\right)^{\prime} w_{t}+\gamma_{t}^{2} L\left(\left\|s_{t}\right\|^{2}+\left\|w_{t}\right\|^{2}\right) \\
\leq & f\left(x_{t}\right)-\gamma_{t} c_{1}\left\|\nabla f\left(x_{t}\right)\right\|^{2}+\gamma_{t} \nabla f\left(x_{t}\right)^{\prime} w_{t}+\gamma_{t}^{2} 2 L c_{2}^{2} \\
& \quad+\gamma_{t}^{2} 2 L c_{2}^{2}\left\|\nabla f\left(x_{t}\right)\right\|^{2}+\gamma_{t}^{2} L\left\|w_{t}\right\|^{2} \\
& \leq f\left(x_{t}\right)-\gamma_{t} \frac{c_{1}}{2}\left\|\nabla f\left(x_{t}\right)\right\|^{2}+\gamma_{t} \nabla f\left(x_{t}\right)^{\prime} w_{t}+\gamma_{t}^{2} 2 L c_{2}^{2}+\gamma_{t}^{2} L\left\|w_{t}\right\|^{2},
\end{aligned}
$$

where the last inequality is valid only when $t$ is large enough so that $\gamma_{t} 2 L c_{2}^{2} \leq c_{1} / 2$. Without loss of generality, we will assume that this is the case for all $t \geq 0$.

Let $\delta>0$ be an arbitrary positive number that will be kept constant until the very end of this proof. Let $\eta$ be a positive constant defined, in terms of $\delta$, by

$$
\eta c_{2}\left(\frac{1}{\delta}+2\right)+\eta=\frac{1}{2 L}
$$

We will partition the set of all times $t$ (the nonnegative integers) into a set $S$ of times at which $\left\|\nabla f\left(x_{t}\right)\right\|$ is "small" and intervals $I_{k}=\left\{\tau_{k}, \tau_{k}+1, \ldots, \tau_{k}^{\prime}\right\}$ during which $\left\|\nabla f\left(x_{t}\right)\right\|$ stays "large." The definition of the times $\tau_{k}$ and $\tau_{k}^{\prime}$ is recursive and is initialized by letting $\tau_{0}^{\prime}=-1$. We then let, for $k=1,2, \ldots$,

$$
\tau_{k}=\min \left\{t>\tau_{k-1}^{\prime} \mid\left\|\nabla f\left(x_{t}\right)\right\| \geq \delta\right\} .
$$

(We leave $\tau_{k}$ undefined if $\left\|\nabla f\left(x_{t}\right)\right\|<\delta$ for all $t>\tau_{k-1}^{\prime}$.) We also let

$$
\begin{aligned}
\tau_{k}^{\prime}=\max \left\{t \geq \tau_{k} \mid\right. & \sum_{i=\tau_{k}}^{t} \gamma_{i} \leq \eta, \text { and } \\
& \left.\frac{\left\|\nabla f\left(x_{\tau_{k}}\right)\right\|}{2} \leq\left\|\nabla f\left(x_{r}\right)\right\| \leq 2\left\|\nabla f\left(x_{\tau_{k}}\right)\right\| \forall r \text { with } \tau_{k} \leq r \leq t\right\} .
\end{aligned}
$$

We say that the interval $I_{k}$ is full if $\sum_{t=\tau_{k}}^{\tau_{k}^{\prime}+1} \gamma_{t}>\eta$. Let $S$ be the set of all times that do not belong to any of the intervals $I_{k}$.

We define a sequence $G_{t}$, used to scale the noise terms $w_{t}$, by

$$
G_{t}= \begin{cases}\delta & \text { if } t \in S, \\ \left\|\nabla f\left(x_{\tau_{k}}\right)\right\|=H_{k} & \text { if } t \in I_{k},\end{cases}
$$

where the last equality should be taken as the definition of $H_{k}$. In particular, $G_{t}$ is constant during an interval $I_{t}$. Note that $G_{t} \geq \delta$ for all $t$.

We now collect a few observations that are direct consequences of our definitions.

(P1) For all $t \in S$, we have $\left\|\nabla f\left(x_{t}\right)\right\|<\delta=G_{t}$.

(P2) For all $t \in I_{k}$, we have

$$
\frac{G_{t}}{2}=\frac{H_{k}}{2} \leq\left\|\nabla f\left(x_{t}\right)\right\| \leq 2 H_{k}=2 G_{t} .
$$

Combining this with (P1), we also see that the ratio $\left\|\nabla f\left(x_{t}\right)\right\| / G_{t}$ is bounded above by 2 . 
(P3) If $\tau_{k}$ is defined and $I_{k}$ is a full interval, then

$$
\frac{\eta}{2} \leq \eta-\gamma_{\tau_{k}^{\prime}+1}<\sum_{t=\tau_{k}}^{\tau_{k}^{\prime}} \gamma_{t} \leq \eta,
$$

where the leftmost inequality holds when $k$ is large enough so that $\gamma_{\tau_{k}^{\prime}+1} \leq \eta / 2$. Without loss of generality, we will assume that this condition actually holds for all $k$.

(P4) The value of $G_{t}$ is completely determined by $x_{0}, x_{1}, \ldots, x_{t}$ and is therefore $\mathcal{F}_{t}$-measurable. Similarly, the indicator function

$$
\chi_{t}= \begin{cases}1 & \text { if } t \in S \\ 0 & \text { otherwise }\end{cases}
$$

is also $\mathcal{F}_{t}$-measurable.

LEMma 2. Let $r_{t}$ be a sequence of random variables with each $r_{t}$ being $\mathcal{F}_{t+1}$ measurable, and suppose that $E\left[r_{t} \mid \mathcal{F}_{t}\right]=0$ and $E\left[\left\|r_{t}\right\|^{2} \mid \mathcal{F}_{t}\right] \leq B$, where $B$ is some deterministic constant. Then, the sequences

$$
\sum_{t=0}^{T} \gamma_{t} r_{t} \quad \text { and } \quad \sum_{t=0}^{T} \gamma_{t}^{2}\left\|r_{t}\right\|^{2}, \quad T=0,1, \ldots,
$$

converge to finite limits (with probability 1 ).

Proof. It is seen that $\sum_{t=0}^{T} \gamma_{t} r_{t}$ is a martingale whose variance is bounded by $B \sum_{t=0}^{\infty} \gamma_{t}^{2}$. It must therefore converge by the martingale convergence theorem. Furthermore,

$$
E\left[\sum_{t=0}^{\infty} \gamma_{t}^{2}\left\|r_{t}\right\|^{2}\right] \leq B \sum_{t=0}^{\infty} \gamma_{t}^{2}<\infty,
$$

which shows that $\sum_{t=0}^{\infty} \gamma_{t}^{2}\left\|r_{t}\right\|^{2}$ is finite with probability 1 . This establishes convergence of the second sequence.

Using Lemma 2, we obtain the following.

Lemma 3. The following sequences converge (with probability 1 ):

(a) $\sum_{t=0}^{T} \chi_{t} \gamma_{t} \nabla f\left(x_{t}\right)^{\prime} w_{t}$;

(b) $\sum_{t=0}^{T} \gamma_{t} \frac{w_{t}}{G_{t}}$;

(c) $\sum_{t=0}^{T} \gamma_{t} \frac{\nabla f\left(x_{t}\right)^{\prime} w_{t}}{G_{t}^{2}}$;

(d) $\sum_{t=0}^{T} \gamma_{t}^{2} \frac{\left\|w_{t}\right\|^{2}}{G_{t}^{2}}$

(e) $\sum_{t=0}^{T} \gamma_{t}^{2} \chi_{t}\left\|w_{t}\right\|^{2}$

Proof. (a) Let $r_{t}=\chi_{t} \nabla f\left(x_{t}\right)^{\prime} w_{t}$. Since $\chi_{t}$ and $\nabla f\left(x_{t}\right)$ are $\mathcal{F}_{t}$-measurable and $E\left[w_{t} \mid \mathcal{F}_{t}\right]=0$, we obtain $E\left[r_{t} \mid \mathcal{F}_{t}\right]=0$. Whenever $\chi_{t}=1$, we have $\left\|\nabla f\left(x_{t}\right)\right\| \leq \delta$ 
and $E\left[\left\|w_{t}\right\|^{2} \mid \mathcal{F}_{t}\right] \leq A\left(1+\delta^{2}\right)$. It follows easily that $E\left[\left|r_{t}\right|^{2} \mid \mathcal{F}_{t}\right]$ is bounded. The result follows from Lemma 2 .

(b) Let $r_{t}=w_{t} / G_{t}$. Since $G_{t}$ is $\mathcal{F}_{t}$-measurable and $E\left[w_{t} \mid \mathcal{F}_{t}\right]=0$, we obtain $E\left[r_{t} \mid \mathcal{F}_{t}\right]=0$. Furthermore,

$$
E\left[\left\|r_{t}\right\|^{2} \mid \mathcal{F}_{t}\right] \leq \frac{A\left(1+\left\|\nabla f\left(x_{t}\right)\right\|^{2}\right)}{G_{t}^{2}} .
$$

Since the ratio $\left\|\nabla f\left(x_{t}\right)\right\| / G_{t}$ is bounded above [cf. observation (P2)], Lemma 2 applies and establishes the desired convergence result.

(c) Let $r_{t}=\nabla f\left(x_{t}\right)^{\prime} w_{t} / G_{t}^{2}$. Note that

$$
\frac{\nabla f\left(x_{t}\right)^{\prime} w_{t}}{G_{t}^{2}} \leq \frac{\left\|\nabla f\left(x_{t}\right)\right\| \cdot\left\|w_{t}\right\|}{G_{t}^{2}} \leq 2 \frac{\left\|w_{t}\right\|}{G_{t}} .
$$

The ratio in the left-hand side has bounded conditional second moment, by the same argument as in the proof of part (b). The desired result follows from Lemma 2.

(d) This follows again from Lemma 2. The needed assumptions have already been verified while proving part (b).

(e) This follows from Lemma 2 because $\chi_{t} w_{t}$ has bounded conditional second moment, by an argument similar to the one used in the proof of part (a).

We now assume that we have removed the zero probability set of sample paths for which the series in Lemma 3 does not converge. For the remainder of the proof, we will concentrate on a single sample path outside this zero probability set. Let $\epsilon$ be a positive constant that satisfies

$$
\epsilon \leq \eta, \quad 2 \epsilon+2 L \epsilon \leq \frac{c_{1} \eta}{48}, \quad 4 L c_{2}^{2} \epsilon \leq \frac{c_{1} \delta^{2} \eta}{48} .
$$

Let us choose some $t_{0}$ after which all of the series in Lemma 3 , as well as the series $\sum_{t=0}^{T} \gamma_{t}^{2}$, stay within $\epsilon$ from their limits.

Lemma 4. Let $t_{0}$ be as above. If $\tau_{k}$ is defined and is larger than $t_{0}$, then the interval $I_{k}$ is full.

Proof. Recall that for $t \in I_{k}=\left\{\tau_{k}, \ldots, \tau_{k}^{\prime}\right\}$ we have $G_{t}=H_{k}=\left\|\nabla f\left(x_{\tau_{k}}\right)\right\| \geq \delta$ and $\left\|s_{t}\right\| \leq c_{2}\left(1+\left\|\nabla f\left(x_{t}\right)\right\|\right) \leq c_{2}\left(1+2 H_{k}\right)$. Therefore,

$$
\begin{aligned}
\left\|x_{\tau_{k}^{\prime}+1}-x_{\tau_{k}}\right\| & \leq \sum_{t=\tau_{k}}^{\tau_{k}^{\prime}} \gamma_{t}\left\|s_{t}\right\|+\left\|\sum_{t=\tau_{k}}^{\tau_{k}^{\prime}} \gamma_{t} w_{t}\right\| \\
& =\sum_{t=\tau_{k}}^{\tau_{k}^{\prime}} \gamma_{t}\left\|s_{t}\right\|+H_{k}\left\|\sum_{t=\tau_{k}}^{\tau_{k}^{\prime}} \gamma_{t} \frac{w_{t}}{G_{t}}\right\| \\
& \leq \eta c_{2}\left(1+2 H_{k}\right)+H_{k} \epsilon \\
& \leq \eta c_{2} H_{k}\left(\frac{1}{\delta}+2\right)+\eta H_{k} \\
& =\frac{H_{k}}{2 L}
\end{aligned}
$$

where the last equality follows from our choice of $\eta$ (cf. (4.5)). Thus,

$$
\left\|\nabla f\left(x_{\tau_{k}^{\prime}+1}\right)-\nabla f\left(x_{\tau_{k}}\right)\right\| \leq L\left\|x_{\tau_{k}^{\prime}+1}-x_{\tau_{k}}\right\| \leq \frac{H_{k}}{2}=\frac{\left\|\nabla f\left(x_{\tau_{k}}\right)\right\|}{2},
$$


which implies that

$$
\frac{1}{2}\left\|\nabla f\left(x_{\tau_{k}}\right)\right\| \leq\left\|\nabla f\left(x_{\tau_{k}^{\prime}+1}\right)\right\| \leq 2\left\|\nabla f\left(x_{\tau_{k}}\right)\right\| .
$$

If we also had $\sum_{t=\tau_{k}}^{\tau_{k}^{\prime}+1} \gamma_{t} \leq \eta$, then $\tau_{k}^{\prime}+1$ should be an element of $I_{k}$, which it isn't. This shows that $\sum_{t=\tau_{k}}^{\tau_{k}^{\prime}+1} \gamma_{t}>\eta$ and that $I_{k}$ is a full interval.

Our next lemma shows that after a certain time, $f\left(x_{t}\right)$ is guaranteed to decrease by at least a constant amount during full intervals.

Lemma 5. Let $t_{0}$ be the same as earlier. If $\tau_{k}$ is defined and larger than $t_{0}$, then

$$
f\left(x_{\tau_{k}^{\prime}+1}\right) \leq f\left(x_{\tau_{k}}\right)-h,
$$

where $h$ is a positive constant that depends only on $\delta$.

Proof. Note that $I_{k}$ is a full interval by Lemma 4. Using (4.4), we have

$$
f\left(x_{t+1}\right)-f\left(x_{t}\right) \leq-\gamma_{t} \frac{c_{1}}{2}\left\|\nabla f\left(x_{t}\right)\right\|^{2}+\gamma_{t} \nabla f\left(x_{t}\right)^{\prime} w_{t}+\gamma_{t}^{2} 2 L c_{2}^{2}+\gamma_{t}^{2} L\left\|w_{t}\right\|^{2} .
$$

We will sum (from $\tau_{k}$ to $\tau_{k}^{\prime}$ ) the terms in the right-hand side of the above inequality and provide suitable upper bounds. Recall that for $t \in I_{k}$, we have $\left\|\nabla f\left(x_{t}\right)\right\| \geq H_{k} / 2$. Thus, also using (4.6),

$$
-\sum_{t=\tau_{k}}^{\tau_{k}^{\prime}} \gamma_{t} \frac{c_{1}}{2}\left\|\nabla f\left(x_{t}\right)\right\|^{2} \leq-\frac{c_{1} H_{k}^{2}}{8} \sum_{t=\tau_{k}}^{\tau_{k}^{\prime}} \gamma_{t} \leq-\frac{c_{1} H_{k}^{2} \eta}{16} .
$$

Furthermore,

$$
\sum_{t=\tau_{k}}^{\tau_{k}^{\prime}} \gamma_{t} \nabla f\left(x_{t}\right)^{\prime} w_{t} \leq 2 H_{k}^{2} \epsilon,
$$

which follows from the convergence of the series in Lemma 3(c) and the assumption that after time $t_{0}$ the series is within $\epsilon$ of its limit. By a similar argument based on Lemma 3(d), we also have

$$
L \sum_{t=\tau_{k}}^{\tau_{k}^{\prime}} \gamma_{t}^{2}\left\|w_{t}\right\|^{2} \leq 2 L H_{k}^{2} \epsilon
$$

Finally,

$$
2 L c_{2}^{2} \sum_{t=\tau_{k}}^{\tau_{k}^{\prime}} \gamma_{t}^{2} \leq 4 L c_{2}^{2} \epsilon
$$

We add (4.8)-(4.11) and obtain

$$
\begin{aligned}
f\left(x_{\tau_{k}^{\prime}+1}\right) & \leq f\left(x_{\tau_{k}}\right)-\frac{c_{1} \eta H_{k}^{2}}{16}+(2 \epsilon+2 L \epsilon) H_{k}^{2}+4 L c_{2}^{2} \epsilon \\
& \leq f\left(x_{\tau_{k}}\right)-\frac{2 c_{1} \eta H_{k}^{2}}{48}+\frac{c_{1} \eta \delta^{2}}{48} \\
& \leq f\left(x_{\tau_{k}}\right)-\frac{c_{1} \eta \delta^{2}}{48} .
\end{aligned}
$$

The second inequality made use of (4.7); the third made use of $H_{k} \geq \delta$. 
Lemma 6. For almost every sample path, $f\left(x_{t}\right)$ converges to a finite value or to $-\infty$. If $\lim _{t \rightarrow \infty} f\left(x_{t}\right) \neq-\infty$, then $\limsup _{t \rightarrow \infty}\left\|\nabla f\left(x_{t}\right)\right\| \leq \delta$.

Proof. Suppose that there are only finitely many intervals $I_{k}$ and, in particular,

$$
\limsup _{t \rightarrow \infty}\left\|\nabla f\left(x_{t}\right)\right\| \leq \delta
$$

Let $t^{*}$ be some time such that $t \in S$ for all $t \geq t^{*}$. We then have $\chi_{t}=1$ for all $t \geq t^{*}$. We use (4.4) to obtain

$$
\begin{aligned}
f\left(x_{t+1}\right) & \leq f\left(x_{t}\right)+\gamma_{t} \chi_{t} \nabla f\left(x_{t}\right)^{\prime} w_{t}+\gamma_{t}^{2} 2 L c_{2}^{2}+\chi_{t} \gamma_{t}^{2} L\left\|w_{t}\right\|^{2} \\
& =f\left(x_{t}\right)+Z_{t} \quad \text { for } t \geq t^{*}
\end{aligned}
$$

where the last equality can be taken as the definition of $Z_{t}$. Using parts (a) and (e) of Lemma 3 , the series $\sum_{t} Z_{t}$ converges. Lemma 1 then implies that $f\left(x_{t}\right)$ converges to a finite value or to $-\infty$. This proves Lemma 6 for the case where there are finitely many intervals.

We consider next the case where there are infinitely many intervals. We will prove that $f\left(x_{t}\right)$ converges to $-\infty$. We first establish such convergence along a particular subsequence. Let $\mathcal{T}=S \cup\left\{\tau_{1}, \tau_{2}, \ldots\right\}$. We will show that the sequence $\left\{f\left(x_{t}\right)\right\}_{t \in \mathcal{T}}$ converges to $-\infty$. To see why this must be the case, notice that whenever $t \in S$, we have $f\left(x_{t+1}\right) \leq f\left(x_{t}\right)+Z_{t}$, where $Z_{t}$ is as in the preceding paragraph and is summable. Also, whenever $t \in \mathcal{T}$ but $t \notin S$, then $t=\tau_{k}$ for some $k$, and the next element of $\mathcal{T}$ is the time $\tau_{k}^{\prime}+1$. Using Lemma $5, f\left(x_{t}\right)$ decreases by at least $h$ during this interval (for $k$ large enough). We are now in the situation captured by Lemma 1 , with $W_{t}=h$ whenever $t=\tau_{k}$. The convergence of the subsequence $\left\{f\left(x_{t}\right)\right\}_{t \in \mathcal{T}}$ follows. Furthermore, since $W_{t}=h$ infinitely often, the limit can be only $-\infty$.

Having shown that $f\left(x_{\tau_{k}}\right)$ converges to $-\infty$, it now remains to show that the fluctuations of $f\left(x_{t}\right)$ during intervals $I_{k}$ cannot be too large. Because the technical steps involved here are very similar to those given earlier, we provide only an outline. In order to carry out this argument, we consider the events that immediately precede an interval $I_{k}$.

Let us first consider the case where $I_{k}$ is preceded by an element of $S$, i.e., $\tau_{k}-$ $1 \in S$. By replicating the first half of the proof of Lemma 4, we can show that $x_{t}-x_{\tau_{k}-1}$ for $t \in I_{k}$ is bounded by a constant multiple of $\delta$ (for $k$ large enough). Since $\left\|\nabla f\left(x_{\tau_{k}-1}\right)\right\| \leq \delta$, this leads to a $c \delta^{2}$ bound on the difference $f\left(x_{t}\right)-f\left(x_{\tau_{k}-1}\right)$, where $c$ is some absolute constant. Since $f\left(x_{\tau_{k}-1}\right) \rightarrow-\infty$, the same must be true for $f\left(x_{t}\right), t \in I_{k}$.

Let us now consider the case where $I_{k}$ is immediately preceded by an interval $I_{k-1}$. By replicating the proof of Lemma 5 (with a somewhat smaller choice of $\epsilon$ ), we can show that (for $k$ large enough) we will have $f\left(x_{t}\right) \leq f\left(x_{\tau_{k-1}}\right)$ for all $t \in I_{k}$. Once more, since $f\left(x_{\tau_{k-1}}\right)$ converges to $-\infty$, the same must be true for $f\left(x_{t}\right), t \in I_{k}$.

According to Lemma $6, f\left(x_{t}\right)$ converges and if

$$
\lim _{t \rightarrow \infty} f\left(x_{t}\right) \neq-\infty
$$

then $\lim \sup _{t \rightarrow \infty}\left\|\nabla f\left(x_{t}\right)\right\| \leq \delta$. Since this has been proved for an arbitrary $\delta>0$, we conclude that if $\lim _{t \rightarrow \infty} f\left(x_{t}\right) \neq-\infty$, then $\lim \sup _{t \rightarrow \infty}\left\|\nabla f\left(x_{t}\right)\right\|=0$, that is, $\nabla f\left(x_{t}\right) \rightarrow 0$.

Finally, if $x^{*}$ is a limit point of $x_{t}$, this implies that $f\left(x_{t}\right)$ has a subsequence that converges to $f\left(x^{*}\right)$. Therefore, the limit of the entire sequence $f\left(x_{t}\right)$, which we have 
shown to exist, must be finite and equal to $f\left(x^{*}\right)$. We have shown that in this case $\nabla f\left(x_{t}\right)$ converges to zero. By taking the limit of $\nabla f\left(x_{t}\right)$ along a sequence of times such that $x_{t}$ converges to $x^{*}$, we conclude that $\nabla f\left(x^{*}\right)=0$.

5. The incremental gradient method revisited. We now provide an alternative view of the incremental gradient method that was discussed in section 4 .

Consider again a cost function $f$ of the form

$$
f(x)=\frac{1}{m} \sum_{i=1}^{m} f_{i}(x)
$$

where each $f_{i}$ is a function from $\Re^{n}$ into $\Re$ that satisfies the Lipschitz condition (4.1). In contrast to the setting of section 4, we now assume that each update is based on a single component function $f_{i}$, chosen at random. More specifically, let $k(t), t=1,2, \ldots$, be a sequence of independent random variables, each distributed uniformly over the set $\{1, \ldots, m\}$. The algorithm under consideration is

$$
x_{t+1}=x_{t}-\gamma_{t} \nabla f_{k(t)}\left(x_{t}\right),
$$

where $\gamma_{t}$ is a nonnegative scalar stepsize. We claim that this is a special case of the stochastic gradient algorithm. Indeed, the algorithm (5.1) can be rewritten as

$$
x_{t+1}=x_{t}-\frac{\gamma_{t}}{m} \sum_{i=1}^{m} \nabla f_{i}\left(x_{t}\right)-\gamma_{t}\left(\nabla f_{k(t)}\left(x_{t}\right)-\frac{1}{m} \sum_{i=1}^{m} \nabla f_{i}\left(x_{t}\right)\right),
$$

which is of the form

$$
x_{t+1}=x_{t}-\gamma_{t} \nabla f\left(x_{t}\right)-\gamma_{t} w_{t},
$$

where

$$
w_{t}=\nabla f_{k(t)}\left(x_{t}\right)-\frac{1}{m} \sum_{i=1}^{m} \nabla f_{i}\left(x_{t}\right)
$$

We now verify that $w_{t}$ satisfies the assumptions of Proposition 3. Due to the way that $k(t)$ is chosen, we have

$$
E\left[\nabla f_{k(t)}\left(x_{t}\right) \mid \mathcal{F}_{t}\right]=\frac{1}{m} \sum_{i=1}^{m} \nabla f_{i}\left(x_{t}\right)
$$

from which it follows that $E\left[w_{t} \mid \mathcal{F}_{t}\right]=0$. We also have

$$
\begin{aligned}
E\left[\left\|w_{t}\right\|^{2} \mid \mathcal{F}_{t}\right] & =E\left[\left\|\nabla f_{k(t)}\left(r_{t}\right)\right\|^{2} \mid \mathcal{F}_{t}\right]-\left\|E\left[\nabla f_{k(t)}\left(r_{t}\right) \mid \mathcal{F}_{t}\right]\right\|^{2} \\
& \leq E\left[\left\|\nabla f_{k(t)}\left(r_{t}\right)\right\|^{2} \mid \mathcal{F}_{t}\right],
\end{aligned}
$$

which yields

$$
E\left[\left\|w_{t}\right\|^{2} \mid \mathcal{F}_{t}\right] \leq \max _{k}\left\|\nabla f_{k}\left(x_{t}\right)\right\|^{2} .
$$

Let us assume that there exist constants $C$ and $D$ such that

$$
\left\|\nabla f_{i}(x)\right\| \leq C+D\|\nabla f(x)\| \quad \forall i, x
$$


(cf. the assumption of Proposition 2). It follows that

$$
E\left[\left\|w_{t}\right\|^{2} \mid \mathcal{F}_{t}\right] \leq 2 C^{2}+2 D^{2}\left\|\nabla f\left(x_{t}\right)\right\|^{2}
$$

so that condition (4.3) is satisfied and the assertion of Proposition 3 holds.

\section{REFERENCES}

[BMP90] A. Benveniste, M. Metivier, And P. Priouret, Adaptive Algorithms and Stochastic Approximations, Springer-Verlag, New York, 1990.

[BeT89] D. P. Bertsekas and J. N. Tsitsiklis, Parallel and Distributed Computation: Numerical Methods, Prentice-Hall, Englewood Cliffs, NJ, 1989.

[BeT96] D. P. Bertsekas and J. N. Tsitsiklis, Neuro-Dynamic Programming, Athena Scientific, Belmont, MA, 1996.

[Ber95a] D. P. Bertsekas, Nonlinear Programming, Athena Scientific, Belmont, MA, 1995.

[Ber95b] D. P. BERTSEKAS, A new class of incremental gradient methods for least squares problems, SIAM J. Optim., 7 (1997), pp. 913-926.

[Bor95] V. S. BoRkAR, Asynchronous stochastic approximations, SIAM J. Control Optim., 36 (1998), pp. 840-851.

[Del96] B. DeLYon, General results on the convergence of stochastic algorithms, IEEE Trans. Automat. Control, 41 (1996), pp. 1245-1255.

[Gai94] A. A. GaIvoronski, Convergence analysis of parallel backpropagation algorithm for neural networks, Optim. Methods Software, 4 (1994), pp. 117-134.

[Gri94] L. GRIPpo, A class of unconstrained minimization methods for neural network training, Optim. Methods Software, 4 (1994), pp. 135-150.

[KuC78] H. J. Kushner AND D. S. Clark, Stochastic Approximation Methods for Constrained and Unconstrained Systems, Springer-Verlag, New York, 1978.

[KuY97] H. J. Kushner And G. YIn, Stochastic Approximation Methods, Springer-Verlag, New York, 1996.

[Lju77] L. LJUng, Analysis of recursive stochastic algorithms, IEEE Trans. Automat. Control, 22 (1977), pp. 551-575.

[LuT94] Z. Q. Luo AND P. Tseng, Analysis of an approximate gradient projection method with applications to the backpropagation algorithm, Optim. Methods Software, 4 (1994), pp. 85-101.

[Luo91] Z. Q. LuO, On the convergence of the LMS algorithm with adaptive learning rate for linear feedforward networks, Neural Comput., 3 (1991), pp. 226-245.

[MaS94] O. L. MANGASARIAN AND M. V. SOlODOv, Serial and parallel backpropagation convergence via nonmonotone perturbed minimization, Optim. Methods Software, 4 (1994), pp. 103-116.

[Pf196] G. Pflug, Optimization of Stochastic Models. The Interface Between Simulation and Optimization, Kluwer, Boston, 1996.

[PoT73] B. T. POLJAK AND Y. Z. TsYPkin, Pseudogradient adaptation and training algorithms, Automat. Remote Control, 12 (1973), pp. 83-94.

[Pol87] B. T. PolJAK, Introduction to Optimization, Optimization Software Inc., New York, 1987.

[TBA86] J. N. Tsitsiklis, D. P. Bertsekas, And M. Athans, Distributed asynchronous deterministic and stochastic gradient optimization algorithms, IEEE Trans. Automat. Control, 31 (1986), pp. 803-812.

[Wal92] H. WALK, Foundations of stochastic approximation, in Stochastic Approximation and Optimization of Random Systems, L. Ljung, G. Pflug, and H. Walk, eds., Birkhauser, Boston, 1992, pp. 53-93. 